\title{
Compression Strength of Saline Water-exposed Epoxy System Containing Fly Ash Particles
}

\author{
KISHORE* \\ Polymer Composites Laboratory \\ Department of Metallurgy \\ Centre for Advanced Studies, Indian Institute of Science \\ Bangalore, 560 012, India \\ P. BARPANDA \\ Department of Ceramic Engineering \\ National Institute of Technology \\ Rourkela, 769008 , India \\ S. M. KuLKARNI \\ Department of Mechanical Engineering \\ National Institute of Technology \\ Surathkal, 575025 , India
}

\begin{abstract}
Epoxy systems with and without different volume fractions of fly ash particulate fillers are made and their absorption due to immersion in saline water maintained at room temperature are established through weight measurements recorded up to $100 \mathrm{~h}$. The 100 -h exposed samples are additionally subjected to compression tests to evaluate the strength. The results show that both neat epoxy and fly ash-bearing composites exhibit differing levels of aqueous medium absorption - it being less in ash-free samples. When the ash content in the system is large, the absorption levels are high. The data further revealed that the unexposed samples generally record an increasing strength value with ash content. However, for the exposed cases, a reversal in trend with the ash content is noticed. Attempts to explain these differing trends are made in this work by analyzing the features observed on the surface of compression-failed samples using fractography employing scanning electron microscopy (SEM).
\end{abstract}

KEY WORDS: epoxy, filler, fly ash, saline water exposure, compression strength, fractography.

\section{INTRODUCTION}

$\mathbf{P}$ OLYMER MATERIALS BY themselves have found extensive use in noncritical products [1]. Such products are used in advanced engineering applications when reinforced with stronger materials. Among the reinforcements, the fibrous variety, whether continuous or 
discontinuous, occupies a key position. Hence, many inorganic and organic fiberreinforced polymer systems have made their entry at various application levels. Because of their large aspect ratio, they yield components, which have anisotropy when the reinforcements are aligned.

In order to secure isotropic properties in composites, reinforcements with near-spherical shapes have been tried [2] e.g., glass microspheres known as microballoons [3,4]. Such man-made reinforcements, though yield attractive mechanical properties, are expensive. Hence, a search for cheaper reinforcements is a key subject that needs the attention of material scientists. One such inexpensive filler to fit the slot is fly ash [5]. Being a byproduct of thermal power plants, its disposal causes considerable environmental problems [6,7]. Hence, there is a need to tap this inexpensive material for possible use with other systems including the polymer-based ones. The present study looks at how this pozzolanic fly ash, when introduced into a thermoset, responds the exposure to an aqueous medium like laboratory-prepared saline water $[8,9]$.

A perusal of literature shows that little attention has been focused on the compressive property of epoxy system in general and filled ones in particular. Fly ash, as it is pozzolanic in nature, forms a cementitious system on absorbing water. Consequently, the mechanical properties change. Hence, in the present study, an attempt to monitor one such mechanical property, namely, the compression strength of epoxy resin containing different amounts of fly ash filler materials has been made. The work also looks at the saline water absorption property of ash-bearing systems by noting the weight measurements at different intervals of time up to $100 \mathrm{~h}$. For a structureproperty correlation, the failure characteristics of such materials on fractured samples were observed under scanning electron microscopy (SEM) following the compression tests.

\section{EXPERIMENTAL PROCEDURE}

\section{Materials}

The matrix system used for the fabrication of composite slabs consisted of a medium viscosity epoxy resin diglycidyl ether of bisphenol-A (DGEBA) (Trade name: LAPOX L-12) and a room temperature curing hardener triethylene tetra-amine (TETA) (Trade name K-6) supplied by Atul India Ltd (Valsad, Gujarat, India). The density of cured neat resin was found to be $1120 \mathrm{~kg} / \mathrm{m}^{3}$. The filler used in composites was obtained from Neyveli Lignite Corporation Ltd, Neyveli, India. This ASTM class 'C' fly ash was found to consist of a mixture of solid and hollow spheres of different sizes with a bulk density of $\approx 900 \mathrm{~kg} / \mathrm{m}^{3}$. The energy dispersive spectroscopy of the fly ash sample revealed the main constituents to be silica and alumina of $\approx 63$ and $26 \%$ by weight, respectively. Other main oxides present were $\mathrm{Fe}_{2} \mathrm{O}_{3}-6.7 \%$ and $\mathrm{TiO}_{2}-2.5 \%$.

\section{Equipment}

An aluminum mold of dimensions $320 \times 170 \times 3 \mathrm{~mm}^{3}$ was prepared for casting the composite slabs. The inner surfaces of the mold were thoroughly covered with a tape on which silicone grease was applied as the releasing agent. 


\section{Fabrication Procedure}

A calculated amount of fly ash was mixed with a measured quantity of epoxy resin plus hardener by gentle stirring for minimizing the formation of air bubbles, whose presence may yield place to the occurrence of defects following curing of cast slabs. The mixture was then slowly decanted into the mold. It was left to cure at room temperature for

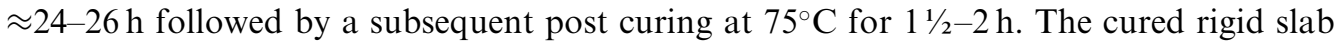
was released from the mold and the edges were trimmed. Using this procedure, epoxy slabs containing 10, 20, and $30 \%$ by volume of fly ash, given as FA10, FA20, and FA30, respectively where the alphabetical abbreviation FA stands for fly ash-bearing composite and the numeral that follows it constitutes the respective volume percent of fillers present in the composite, in this work, were cast. In addition to this, slabs were also cast with neat epoxy resin system (NE) (i.e., without any reinforcing ash particles) containing the required amount of hardener. From the cast slabs, test samples of size $12.5 \times 3 \times 3 \mathrm{~mm}^{3}$ were sectioned for following first the water ingression and then for evaluating the compression strength of the resulting material.

\section{Saline Water Exposure}

To study the absorption of aqueous medium due to immersion, laboratory-prepared saline water was employed. For this, $4 \mathrm{wt} \%$ of $\mathrm{NaCl}$ solution was prepared in a beaker. While one batch of samples of each composition was kept as such (i.e., without exposure), the other was immersed in this medium in the beaker maintained at an ambient temperature. The former category formed the unexposed set while the latter the exposed ones, both the sets being subjected to compression tests later on. For monitoring the weight change at periodic intervals of time, the appropriately coded samples, whose dry weights were noted prior to immersing in water, were withdrawn and wiped dry using an absorbent material. They were weighed accurately, at ambient conditions, using an electronic balance in order to note the difference consequent to saline water exposure. Weight readings were taken at regular intervals of time (Figure 1). Every time, five samples were used for weight data gathering and the average value thus determined was used for data analysis. This procedure of aqueous medium data acquisition was repeated for each of the compositions (i.e., NE, FA10, FA20 and FA30) cast in this work.

\section{Compression Testing}

Compression testing was performed on the test coupons with the help of DARTEC9500, a servohydraulic microprocessor-based computer-controlled testing machine. The loading direction coincided with the length of the specimen and was perpendicular to the $3 \times 3 \mathrm{~mm}^{2}$ cross section. The machine crosshead was programmed to apply the compression load at a constant strain rate of $0.01 \mathrm{~s}^{-1}$ throughout the test by using the built-in software. The test was terminated automatically in case the sample fractured or loaded to $50 \%$ strain, whichever occurred earlier. Often it was observed that the samples fractured much before reaching $50 \%$ strain. From the load-stroke graphs, the compressive strength was determined. A minimum of five samples, as stated earlier, were used for compression tests on any similarly processed samples of different compositions. 


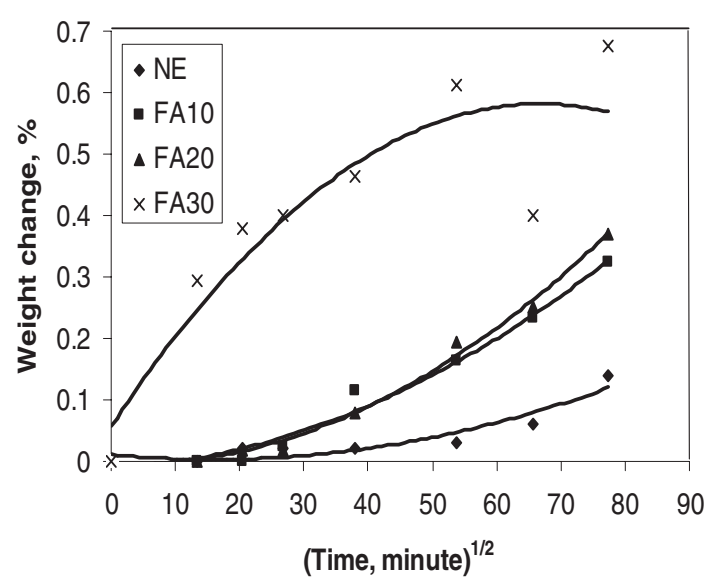

Figure 1. Weight changes in ash-free and ash-bearing epoxy composites subjected to saline water exposure.

\section{Fractography}

Fractographic analysis was performed on the fractured samples using a JEOL make JSM 840A model scanning electron microscope (SEM). Prior to the test, the fractured surfaces of the samples were gold coated in an ion sputtering unit (JEOL make-JFC 1100E model) for over $6 \mathrm{~min}$. The fractured surfaces were observed at suitable magnifications. The fractographs revealing the respective characteristic details of each sample were taken after suitable adjustments in probe current, voltage, image contrast and brightness, working distance, and tilting at various angles. In this manner, photographs were taken on compression-failed, unexposed and exposed samples.

\section{RESULTS AND DISCUSSION}

Figure 2 displays the variation in strength data with respect to the ash content tested in unexposed condition. From the plot, it is apparent that a general upward trend for strength is recorded as ash content in the matrix increases.

To understand how the ash contributes to strength through a resistance offered to deformation, the structural features, as stated earlier, are studied using SEM on these compression-failed samples. Whereas the unexposed (i.e., dry) and unreinforced (NE) sample shows a typical river pattern (Figure 3), the fly ash-bearing ones reveal the influence the ash particles bring onto the failure features. Thus the $10 \%$ case, showing a higher strength than the unreinforced case (Figure 2), displays the resistance offered by the ash in the form of deformation bands which are present as curvilinear features marked 1-2-3-4 toward the center top of the micrograph (Figure 4). It is of interest to note that the band marked 2 has at least three different sizes of ash particles that are in its path resisting the flow of matrix. A careful perusal of this micrograph illustrates the existence of faint smaller bands between the bands numbered 2 and 3 . These represent the resistance to deformation offered by the ash particles (having a modulus $73 \mathrm{GPa}$, which is about 49 times that of the matrix epoxy material) [10] and consequent enhancement of the strength values. The voids marked ' $\mathrm{V}$ ' present around an ash 


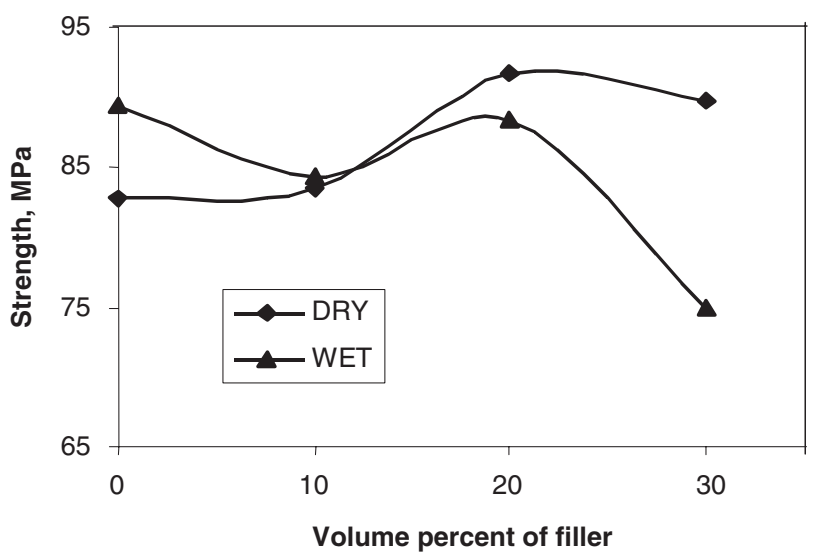

Figure 2. Strength of unexposed and exposed samples containing varying amounts of fly ash.

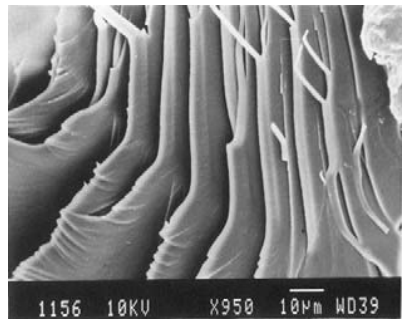

Figure 3. Typical river pattern in unexposed neat epoxy (NE) sample.

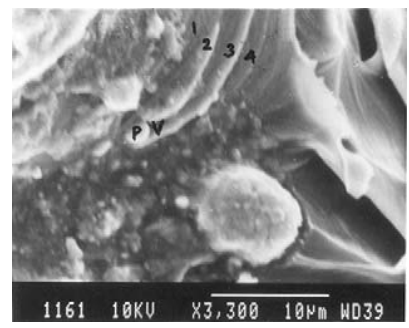

Figure 4. Curvilinear deformation bands in FA10 sample.

particle ' $\mathrm{P}$ ' helps in triggering failure (Figure 4). The quasi-cleavage pattern in this sample is very well depicted in Figure 5, under low magnification. For the $20 \%$ ashbearing samples, which show a high strength (Figure 2), the features recorded are different. Thus Figure 6 shows a stepped appearance in the center of the micrograph and a bow-like feature (marked by an arrow) toward the left top corner. A careful analysis of this bow-like feature at a higher magnification shows (Figure 7) quite a few ash particles marked 1,2, and 3 or a, b, and c or A lying in their paths and responsible for the resistance offered by the system. Of interest to note in this figure is the way the particles get distributed as a network. It is this network $[11,12]$ and the resultant extent 


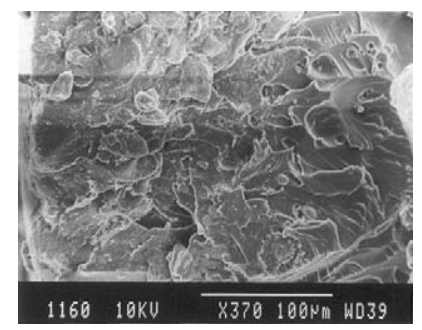

Figure 5. Quasi-cleavage pattern in FA10 sample.

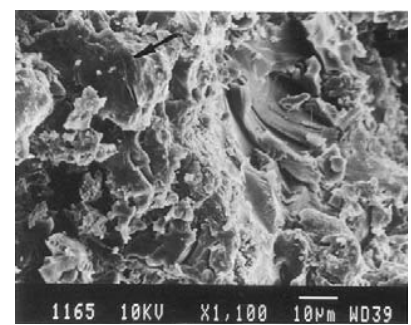

Figure 6. Stepped appearance and bow-like features of FA20 sample.

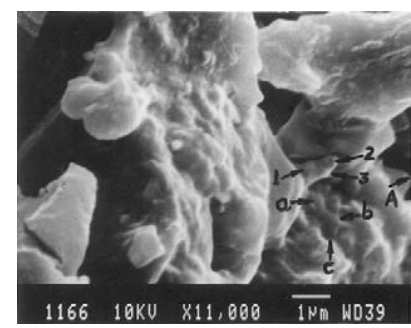

Figure 7. Typical particle network in FA20 sample.

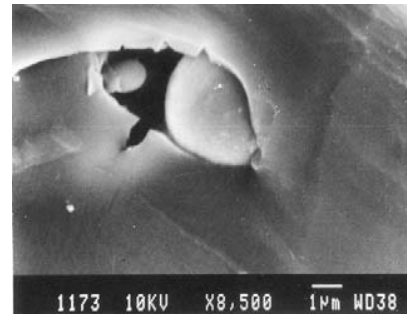

Figure 8. Interfacial debonding and crack opening near two closely posited ash particles in FA30 sample.

of strain energy overlap [13] that aid this sample in recording higher strength than the ash-free one. As regards the $30 \%$ case, the closeness of the particles and consequently the ease with which the interface separation leading to failure occur are responsible for a small drop in strength. These features are illustrated in Figures 8 and 9, where consequent to the two ash particles being posited closely, the crack starts to emerge out 


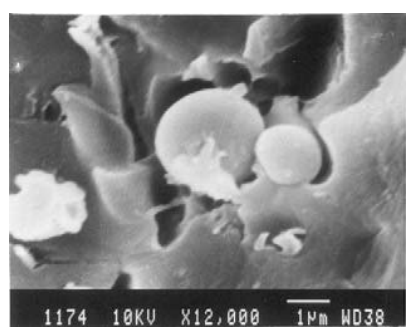

Figure 9. Interfacial debonding and the paths of the crack around two ash particles in FA30 sample.

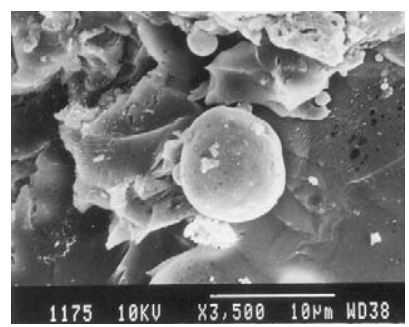

Figure 10. Cleavage facet on compression-failed FA30 sample.

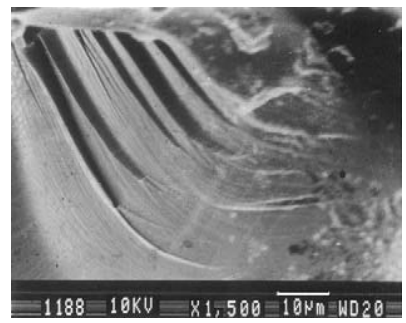

Figure 11. Shear deformation marks in medium-exposed NE sample.

of such a region. This causes an earlier onset of failure. The cleavage facet seen around the ash particle (Figure 10) is a consequence of the brittle fracture process.

Coming to the features seen in the medium-immersed and compression-tested case, Figure 2 records the strength data for this condition. Here, a general drop in strength can be seen. The decrement is large for the highest ash-bearing samples i.e., 30\% FA. To explain why such trends occur, the scanning electron micrographs observed on both ash-free and ash-bearing samples are presented. Figure 11 illustrates the details in NE (i.e., without ash fillers) system following water ingression and compression tests. Compared to the dry case, these unreinforced systems in the wet case show a higher strength (Figure 2). As regards the microscopic feature, the distinct change (vis-à-vis Figure 3 discussed earlier for the dry case) brought about by the aqueous medium ingression is highlighted in Figure 11. The river pattern, clearly seen in the dry samples, vide Figure 3, yields place to shear mode of deformation in medium-exposed condition, Figure 11. Consequently, the strength recorded here is higher compared to that of the dry case (Figure 2). Water is known to plasticize the epoxy system and expand the matrix following ingression. This plasticized material acts as a crack arrester and consequently the failure is dominated 
by shear process unlike the cleavage or river pattern feature exhibited (Figure 3) by unreinforced, unexposed (i.e., dry) samples.

As regards the difference in performance following ingression in ash-bearing samples, it can be traced to the interface separation occurring around ash particles in these samples (Figure 12). Here, the crack involving the interface separation of three closely posited fly ash particles in FA10 can be seen. The expansive nature of first the fly ash particles, (as fly ash is known to be pozzolanic in nature) [14] and then the matrix material, due to aqueous medium ingression, is offset by the interfacial separation factor. Consequently, the strength difference between dry and wet becomes insignificant. For the $20 \%$ case, which showed the highest value for the dry case, it shows a value, which is lower in medium-ingressed condition (Figure 2). This change in response can be traced to the interface separation factor, mentioned earlier and depicted in Figure 13. The difference in response between Figure 6 (dry) and Figure 14 (wet) for the same FA20 case is very obvious. In the former, the interface separations around ash particle are less markedly seen while in the latter, it is distinctly brought out. To highlight that the interfacial debonding between filler and matrix following water ingression is responsible for the

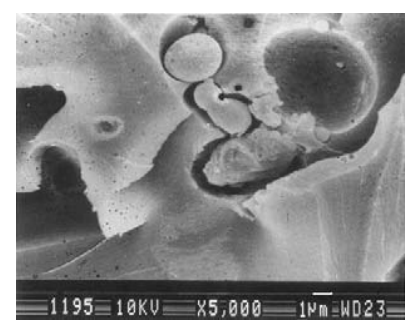

Figure 12. Interface separation of FA20 sample subjected to medium ingression.

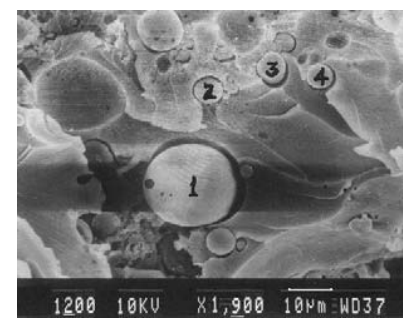

Figure 13. The debonds around ash particles marked 1-4 in FA20 medium-exposed sample.

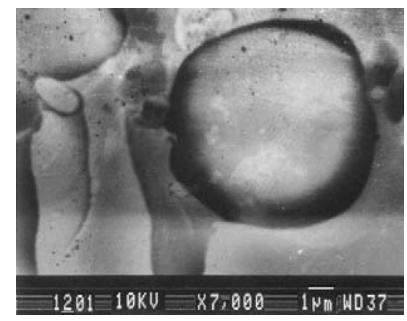

Figure 14. Interfacial separation in FA20 medium-exposed sample. 


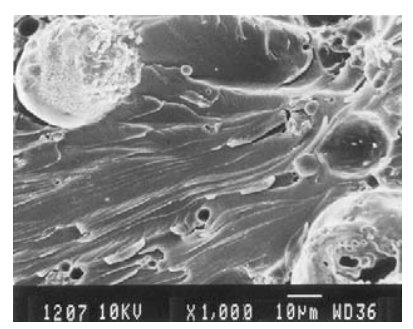

Figure 15. The fracture features in FA30 medium-exposed sample.

observed difference, in Figure 13, four ash particles (marked 1-4) having clear interface debonding around them are shown. Compared to Figure 7, the debonds here are very distinct. Thus the role of water ingression and its influence in the region between the filler and the matrix in the form of appearance of debonds is very distinctly brought out in this study. Coming to the $30 \%$ case, the wet samples show a much steeper drop (Figure 2). To explain this, scanning electron microscopic examination of the water-ingressed sample of FA30 was carried out, which revealed that the ash particles besides showing interface debonds, discussed earlier and shown in the top left corner of Figure 15 for this $30 \%$ case, have the features of shear deformation. But the noticeable difference is that the ash particle appears affected by the deformation process. Thus while the top left shows debris distributed on it, the bottom right shows punctured holes within the particles. It is this difference, which causes the resistance to deformation to matrix flow to be quite different compared to that of the cases having lower ash contents.

\section{CONCLUSIONS}

(i) The larger the fly ash content in the polymer, the higher the extent of moisture ingression.

(ii) Whereas strength in dry condition increases with initial addition of ash particles, those for the medium ingressed show a decrement. This decrease is quite large for the higher fly ash-bearing samples. Interface debonding occurring around ash particles, seen in SEM, has been traced to be the cause for such changes in response in the wet samples vis-à-vis the dry ones.

\section{ACKNOWLEDGMENTS}

The authors wish to thank Mr. S. Sasidhara, Mr. Gurulinga, and Mr. D. Mollaiah of the Department of Metallurgy for the help rendered by them during the testing and inspection of samples. The first author (Kishore) wishes to express his gratitude to the Chairman, Department of Metallurgy for allowing the use of facilities. The second author (P. Barpanda) wishes to express his gratitude to the concerned authorities at IISc for providing fellowship under the 'Young Engineering Fellowship Programme - 2001' scheme. The third author (S. M. Kulkarni) would like to thank the authorities at NITK, Surathkal for granting permission to do the work at IISc. 


\section{REFERENCES}

1. Seymour, R. B. (1990). Engineering Polymer Source Book, Mc-Graw Hill Pub. Co., New York.

2. Ryan, B. and Tardy, B. (1978). Katz, H. S. and Milewski, J. V. (eds), Handbook of Fillers and Reinforcements for Plastics, pp. 301-316, Van Nostrand Reinfold Co., New York.

3. Corigliano, A, Rizzi, E and Papa, E (2000). Experimental Characterization and Numerical Simulations of a Syntactic-foam/Glass-fibre Composite Sandwich, Compos. Sc. and Tech., 60: 2169-2180.

4. Kim, H. S. and Khamis, M. A. (2001). Fracture and Impact Behaviours of Hollow Micro-Sphere/Epoxy Resin Composites, Composites: A, 32: 1311-1317.

5. Torrey, S. (1978). Coal Ash Utilisation - Fly Ash, Bottom Ash and Slag, Noyes Data Corporation, NJ, USA.

6. Mohapartra, R. and Rajagopala Rao, J. (2001). Some Aspects of Characterization, Utilization and Environmental Effects of Fly Ash, J. Chem. Technol. Biotechnol., 76(1): 9-26.

7. Ulaganathan, M. and Pillai, T. M. (1996). Proceedings of National Seminar on Fly ash Utilisation, Neyvelli, India, p. 43.

8. Adams, R. D. and Singh, M. M. (1995). The Effect of Immersion in Sea Water on the Dynamic Properties of Fibre-reinforced Flexibilised Epoxy Composites, Composite Structures, 31(2): 119-127.

9. Gellert, E. P. and Turley, D. M. (1999). Sea Water Immersion Ageing of Glass-fibre Reinforced Polymer Laminates for Marine Applications, Composites- $A$, 30(11): 1259-1265.

10. Shackelford, J. F. and Alexander, W. (1992). The CRC Materials Science and Engineering Handbook, pp. 436438, CRC Press, Boca Raton, FL, USA.

11. Milewski, J. V. (1978). Katz, H. S. and Milewski, J. V. (eds), Handbook of Fillers and Reinforcements for Plastics, Van Nostrand Reinfold Co, New York.

12. Kulkarni, S. M. (2002). Processing, Microstructural and Mechanical Behavioural Aspects of Fly Ash-Epoxy Composites, PhD Dissertation, Indian Institute of Science, India.

13. Zellen, R. (1978). Physics of Amorphous Solids, pp. 183-188, John Wiley, New York.

14. ASTM C 618 Standard Specification for Coal Fly Ash and Raw or Calcined Natural Pozzolan for use as a Mineral Admixture in Portland Cement Concrete, American Society for Testing of Materials, Philadelphia, USA. 